http://jmscr.igmpublication.org/home/ ISSN (e)-2347-176x ISSN (p) 2455-0450 crossref DOI: https://dx.doi.org/10.18535/jmscr/v9i10.21

\title{
Retrospective Clinical and Epidiemological Evaluation of Snake Bite Cases in Kishanganj District of Rural Bihar
}

\author{
Authors \\ Dr Sourav Chattopadhyay ${ }^{1}$, Dr Abhinav Chaudhary ${ }^{2 *}$, \\ Dr Sumit Singh Kaushal ${ }^{3}$, Dr Sandeep Bhardwaj ${ }^{4}$, Dr Shubham Shukla ${ }^{5}$, \\ Dr Aniket Sinha ${ }^{6}$, Dr Suman Kumar Singh ${ }^{7}$ \\ ${ }^{1}$ Associate Professor, Dept. of General Medicine, M.G.M., Medical College \& L.S.K. Hospital, Kishanganj, \\ Bihar
}

${ }^{2,3,4,5,6,7}$ Resident, Dept. of General Medicine, M.G.M. Medical College \& L.S.K Hospital, Kishanganj, Bihar *Corresponding Author

Dr Abhinav Chaudhary

\begin{abstract}
Introduction: Snake envenoming is a significant and neglected global public health issue causing multiple potentially life-threatening toxin mediated clinical syndrome. Snakebite is now recognized as a Neglected Tropical Disease by the World Health Organization. ${ }^{[1]}$

WHO estimates about 5 million people are bitten each year by poisonous snakes which results in 2.5 million envenomations, at least 100000 deaths, and 300000 amputations and other permanent disabilities. Majority of snakebite induced deaths occur in Asia and Sub-Saharan Africa. The mortality due to venomous snakebite in India is estimated between 35000-50000 per annum, which is the highest in the world ${ }^{[1]}$.

Bihar, Jharkhand, Madhya Pradesh, Odisha Uttar Pradesh, Andhra Pradesh,Telangana, Rajasthan and Gujarat are eight states defined as high burden states for snake bite. ${ }^{[2]}$

The study has been done in MGM Medical college and LSK Hospital at Kishanganj District under Purnia division having predominant rural population engaged in farming practices.]

The primary objective being to study the demographics, analyse the clinical and epidemiological profile and outcome of snake bite cases by retrospective evaluation of hospital records from May 2019 to May 2020.

Snake venom may contain twenty or more toxins. Most of them are enzymes, non-enzyme peptide toxins and non-toxic proteins. ${ }^{[1]}$ The potency and composition of toxin depends on diet, geographical location and climatic condition of the snakes habitat. Cobras, Kraits, Russell's viper and Saw Scale vipers are the commonly found venomous species of snakes responsible for maximum morbidity and mortality in India.

Bihar witnesses deaths of around 4,500 people every year due to snake bites, majority of them are from extremely poor backgrounds and stands as the third in the country with the largest number of deaths caused by snakebites in India ${ }^{[3]}$

Methods: Data extracted from medical records of snakebite victims admitted in the Medicine ward and Intensive care at Mata Gujri Memorial Medical College and LSK Hospital during the period extending from May 2019 to May 2020 was subjected to retrospective analysis to describe Clinico-epidemiological profile
\end{abstract}


and management of snake bite cases. Age /gender distribution, site of envenomation, Time lag of presentation seasonal trends of snake bite cases, duration of hospital stay and complications have been described in the study. Proportion of envenomation and dry bite was assessed as well as case fatality rate computed from data collected.

Statistical Analysis: Statistical analysis was done using SPSS software.

Results: Out of 440 cases analysed from hospital records from May 2019 to May 2020. 61\% were male predominantly engaged in farming and agricultural activities. Foot and ankle followed by area below knee joint was most common site of bite both in male and female patients. Maximum number of cases occurred in August which also has highest rainfall followed by October which corresponds to harvesting season of paddy crop in Bihar.

Most cases presented within mean time of two hours of snake bite whereas six cases presented after 7 to 10 days with psychiatric symptoms. The proportion of dry bite and bite with nonpoisonous snakes was higher as compared to envenomation being (58\%) and (42\%) respectively.

Among the envenomation cases majority were neurotoxic(44\%), hematotoxic (37\%), and (19\%) cases of local envenomation.

Mean duration of hospital stay was five days. Long term complications like necrosis, amputation, renal failure was identified in 42 patients most of whom presented beyond six hours of snake bite.

Case fatality rate was $3.6 \%$ (16\440).

Conclusion: Snake bite is a preventable public health problem and the morbidity and mortality associated can be minimized by increasing public awareness and timely intervention. As maximum number of bite cases are accidental or provoked and during outdoor farming activities use of rubber boots and protective clothing helps to reduce the incident.

Antisnake venom should be developed using venom from the same region, adequate infrastructure in terms of dedicated centres in each district and decentralization of ASV production is need of the hour.

Keywords: Snake bite, Envenomation, Poisonous snakes, Anti snake venom.

\section{Introduction}

Snake bite is a neglected public health problem in the world and one of the major causes of mortality and morbidity in many areas, particularly in the rural tropics. It also poses substantial economic burdens on the snake bite victims due to treatment related expenditure and loss of productivity ${ }^{[4]}$

Of the total population of Bihar state, around 88.71 percent live in the villages of rural areas. In actual numbers, males and females were $48,073,850$ and 44,267,586 respectively.

Total population of rural areas of Bihar state was $92,341,436.1$ population of rural areas of Bihar state was $92,341,436 .^{[5]}$

https://www.census2011.co.in]

Underestimation of snakebite mortality can be a result of snake bite victims dying en route to health care facilities, and many others do not report to the health centers because they were cured leading to underestimation of morbidity. Nevertheless, complications of snake bite (amputations, renal failure, coagulopathy, neurologic deficits) are common.
Snakebite morbidity is more likely to be underestimated than mortality because death is a less frequent outcome and probably better reported than envenoming.

Snake venom may contain twenty or more toxins. Most of them are enzymes, non-enzyme peptide toxins and non-toxic proteins. ${ }^{[1]}$

Snake bite is not only a medical emergency but is also associated with long term complications. Study done by Jayawardana et $\mathrm{al}^{[6]}$ in Bangladesh shows "migraine-like-syndrome" characterized by headache vertigo, and photosensitivity to sunlight, musculoskeletal disorders such as pain, local swelling, muscle weakness, deformities, contractures, and amputation, visual impairment, Acute kidney injury, psychological distress, hemiplegia, facial nerve palsy, paresthesia over bite site and nonspecific somatic symptoms such as abdominal colic, chest tightness, wheezing, excessive hair loss, and lassitude with body aches following the bite. 
According to a study by Kasturiratne et al published in 2008 shows that globally, at least 421,000 envenomings and 20,000 deaths occur each year due to snakebite. ${ }^{[7]}$

Bihar, Jharkhand, Madhya Pradesh, Odisha, Uttar Pradesh, Andhra Pradesh, Telangana, Rajasthan and Gujarat are eight states defined as high burden states for snake bite. ${ }^{[2]}$ Bihar has the third largest number of snakebite deaths of any state in India.

The four venomous snake species responsible for causing the greatest number of medically significant human snake cases on the Indian Subcontinent collectively referred to as the Big Four $^{[8]}$ [Indian J Med Res.2012 Jul; 136(1)] Are as follows:

1. Common krait, Bungarus caeruleus

2. Russell's viper, Daboia russelii

3. Indian saw-scaled viper, Echis carinatus

4. Indian cobra, Naja naja

In our study Cobra and Krait bites were more common.

The cobra and krait venoms are neurotoxic and cardiotoxic. Viper venom is vasculotoxic and has severe necrotizing local effects. ${ }^{[1]}$

Major determinants of survival and morbidity according to our study was the time of presentation often hindered by mode of transportation and practice of seeking traditional methods of evenomation practiced by snake charmers and quacks rampant in rural areas.

Non availablity of Polyvalent anti-snake venom (ASV), high cost per vial and large number of such vials required for neutralization of venom were other contributing factors. Each vial of Polyvalent anti-snake venom (ASV) serum contains $10 \mathrm{~mL}$, and each $1 \mathrm{~mL}$ neutralizes $0.6 \mathrm{mg}$ cobra, $0.6 \mathrm{mg}$ Russell's viper, $0.45 \mathrm{mg}$ common krait, and $0.45 \mathrm{mg}$ saw- scaled viper venoms.

\section{Methods}

\section{Study Design}

It is a Hospital record based retrospective, observational, epidemiological study. The study was carried out after due approval from institutes ethical committee.

\section{Study Duration}

Study was conducted for a period of one year between May 2019 to May 2020.

\section{Study Settings}

This study was carried out by the Department of Medicine, in Mata Gujri Memorial Medical College and Lions Seva Kendra Hospital, Kishanganj.

Kishanganj district is located in extreme north of Bihar, 55m above the sea level and enjoys a humid subtropical climate.

The hospital caters to the health needs of rural and urban communities of the city and also the neighboring districts of West Bengal.

\section{Sample Population}

Snake bite cases fulfilling the inclusion/ exclusion criteria who presented in emergency deptt and admitted in General Medicine wards or intensive care unit of Mata Gujri Memorial Medical College and Lions Seva Kendra Hospital were included during the study period of one year. A total of 440 snake bite cases were included in the study.

\section{Inclusion Criteria}

A. Case with history of snake bite irrespective of the envenomation status.

B. Patients with one or more symptoms of clinical manifestations of snake bite like local swelling, hemorrhage, blisters formation, vomiting, abdominal pain and regional lymphadenopathy, neurological manifestations.

\section{Exclusion Criteria}

A. Patients with bites other than snake bites

B. Patient with a known history of bleeding disorders, malignancies, neurological disorders, endocrine disorders, underlying renal and liver disorders.

C. Chronic alcoholics and drug addicts.

D. Patients on anticoagulant drugs.

E. Brought dead patients with alleged history of poisonous bite. 


\section{Sampling Size and Sample Design}

The records of the 440 snakebite victims who were admitted as in patients in the hospital during the period were extracted from the medical records department. This department uses the ICD-10 system for the Classification of diseases. Clinical data including age wise distribution, gender, occupation of the victims, the site of bite, Seasonal trends, time between bite and presentation, clinical manifestations, complications and mortality outcomes, were extracted from the case files.

\section{Results}

Among the 440 cases studied $80.2 \%$ were from rural background whereas $19.8 \%$ were living in an urban setup.

The study showed male preponderance with (268\440) $61 \%$ of snake bites victims being males engaged in farming and outdoor activities. Female victims 39\% mostly were from rural background living in thatched houses close to the habitat of snakes.

Maximum number of bites $57.2 \%$ (252\440) were over exposed areas of ankle and foot, followed by leg and thigh in $17.7 \%$ of cases. Minimum number of bites occurred over chest and abdomen (3.6\%).Majority of bites were accidental.

Most of these bites were dry bites or due to nonpoisonous snakes (58\%) and the patients presented with tachycardia, chest tightness, anxiety, sweating most attributed to sympathetic over activity. Among the $42 \%$ (185) cases of envenomation $55 \%$ were neurotoxic, $30 \%$ cases of local envenomation and remaining vasculotoxic.

A large number of these cases 61\%(312\440) presented to the casualty within two hours of the bite and were promptly given ASV as and when indicated.18\% presented between two to six hours, $10 \%$ between 12 to 24 hours whereas a small minority of $1 \%(61440)$ presented beyond 24 hours.

Clinical presentation with swelling, blistering or necrosis at the site of the bite and its extension.
Hypotension, cardiogenic shock, Haemorrhage Myoglobinuria, Laboratory evidence of coagulation defect or bedside Positive 20 minute whole blood clotting test was observed.

Neuroparalytic manifestations like Ptosis, External ophthalmoplegia, weakness of neck muscles and bulbar muscles, blurring of vision, perioral paraesthesia observed. Arrhythmias, bradycardia, tachycardia, were the cardiac manifestations encountered. Maximum number of cases occurred in middle age group followed by children.

Case fatality rate was 3.6\%(161440).

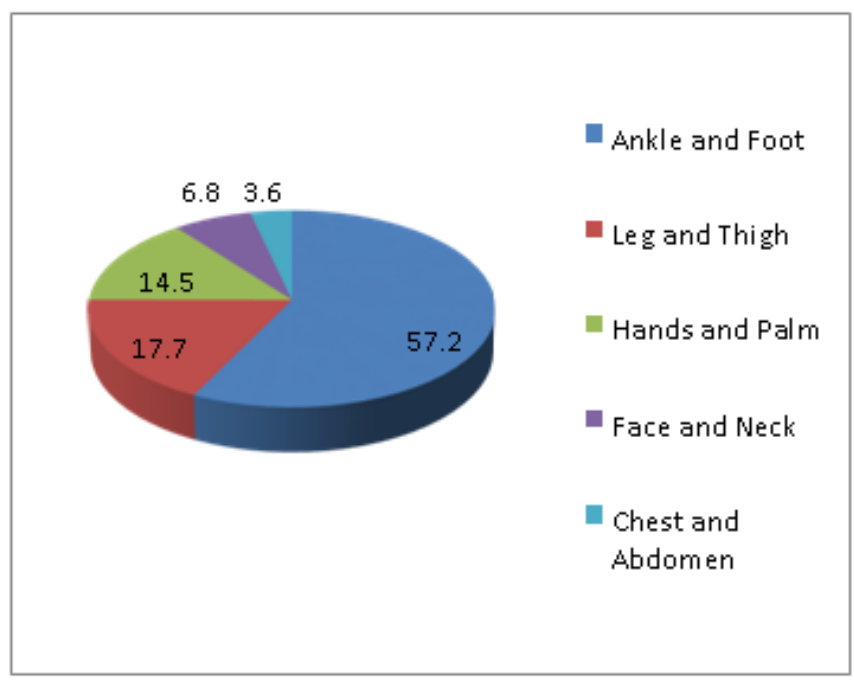

Figure 1 Site of Bite

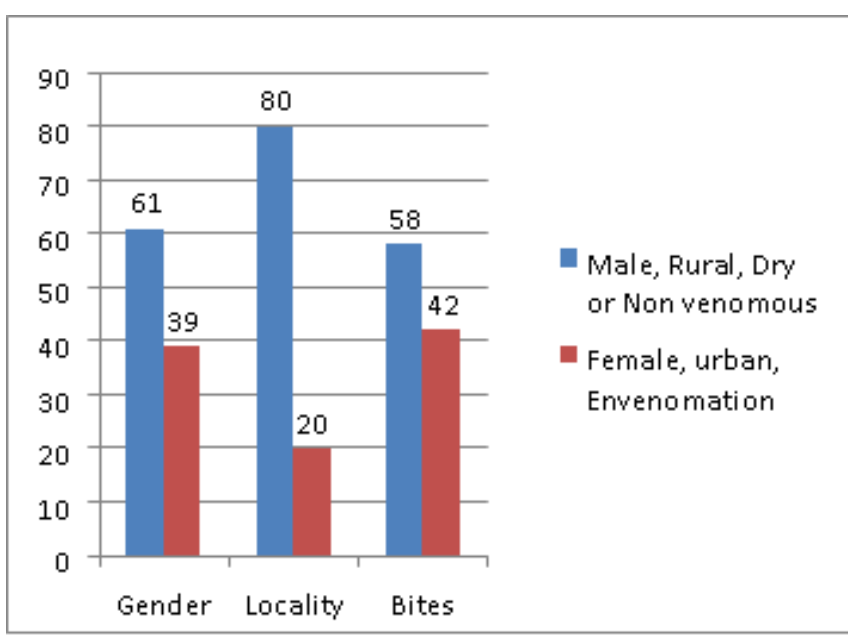

Figure 2 Distribution of cases based on Gender, Locality and Type of bite 


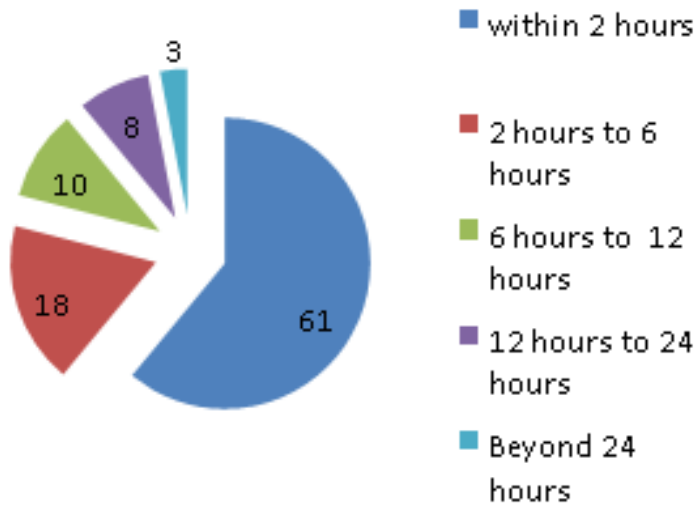

Figure 3 Hours to treatment

\section{Discussion}

Retrospective pattern is the most commonly employed observational study design used for evaluation of snake bite cases this points towards the importance of maintenance of the hospital records and notification of diseases following the ICD 10 system of classification.

We had screened around three thousand medical records to extract the sample size of 440 patients.

Our Study benign a time bound study carried over a period of one year the sample size was not a pre determinant for the study.

Although, Urbanization has led to decreased number of snakes and snakebite cases it is also a factor responsible for destruction of their natural habitat and the migration of these species towards human settlements.

The World Health Organization (WHO) estimates that 81,000-138,000 people die each year from snakebites worldwide, and about three times that number survive and but are left with amputations and permanent disabilities. ${ }^{[2]}$ In 2017 , the WHO included snakebite envenoming in the priority list of neglected tropical diseases and launched in 2019 a strategy for prevention and control of snakebite, aiming to halve the numbers of deaths and cases of serious disability by 2030 as compared to 2015 baseline. ${ }^{[2]}$

Bihar is the state with the third largest number of snakebite deaths per year in India. ${ }^{[10]}$

A prospective hospital based study by

Longkumer et al in East champaran district of Bihar showed neurotoxic poisoning is maximum among the cases of envenomation and Cobra and krait being most commonly identified poisonous species identified. ${ }^{[10]}$ Our study has similar conclusion.

Almost all studies have shown male preponderance although percentage having a significant margin as example Sharma et al showed $73 \%$ to be male victims, Brunda et al and Chattopadhyay et al had a male percentage of $76 \%$ and $63 \%$ respectively. Our study has $61 \%$ of male victims. However a study by Rahman $\mathrm{Ra}$ et al in rural Bangladesh shows almost equal sex distribution of snake bite cases.

According to the study by Longkumer et al maximum number of bites was in children aged below fifteen years which is not in accord to our study, results of which shows that maximum cases occured in middle aged indivisuals followed by children.

Majority of the studies report the most common site of bite to be ankle and foot, in our study around $60 \%$ of the bites were over ankle and foot.

Study by Suraweera et al estimates the risk of an Indian dying from snakebite before age 70 is about 1 in $250 .^{[2]}$

The case fatality rate computed by our study is 3.6 percent.

\section{Conclusions}

Bihar is third in number accounting for snake bite cases preceded only by Andhra Pradesh and Uttar Pradesh.

As highlighted maximum incidence, morbidity and mortality of snake bites occur in rural region adequate and equipped rural health centers with expertise in management of envenomation needs to be developed.

Our study highlights the need for early intervention post bite as the rate of recovery was improved when the patients were administered anti snake venom within 1 hour of the bite.

Role of timely referral, availability of appropriate transportation, education of the local population, discouraging reliability on traditional practices and encouraging proper reporting of cases will ensure 
reduction in mortality and morbidity associated with snake bite.

Accessibility of Life Support Measures like Ventilators for Respiratory Failure and hemodialysis units for AKI at the grass root level would prevent the time lost in referral.

Decentralising ASV production with Anti snake venom being developed using venom from the same region would be a breakthrough in this regard.

The Haffkine Institute For Training, Research and Testing in Parel has proposed the development of a national venom research centre for better diagnosis and treatment of snake bites in India. ${ }^{[1]}$ In addition to this, Haffkine is also planning to conduct a countrywide venom mapping and improving the availability of anti-snake venom both of which would be a major breakthrough.

\section{References}

1. Col SR Mehta,Wg Cdr VK Sashindran; Clinical Features And Management Of Snake Bite; MJAFJ. VOL 58. NO.3. 2002

2. Suraweera et al;Trends in snakebite deaths in India from 2000 to 2019 in a nationally representative mortality study eLife 2020; 9:e54076.

DOI: https://doi.org/10.7554/eLife.54076

3. THE INDIAN EXPRESS "Over 4500 people die of snakebites every year in Bihar" Published: 24th November 2019.

4. Rahman R, Faiz MA, Selim S, Rahman B, Basher A, et al; (2010) Annual Incidence of Snake Bite in Rural Bangladesh. PLoS Negl Trop Dis 4(10):e860. DOI:10.1371/journal.pntd.0000860 https://www.census2011.co.in

5. Jayawardana et al;Long-term health complications following snake envenoming; Journal of Multidisciplinary Healthcare 2018:11 279-285.

6. Kasturiratne A, Wickremasinghe AR, de Silva N, Gunawardena NK, Pathmeswaran A, et al. (2008) Estimating the global burden of snakebite: A literature analysis and modelling based on regional estimates of envenoming and deaths. PLoS Med $5(11)$ : e218. doi:10.1371/journal.pmed.0050218

7. Warell et al; Indian J Med Res.2012 Jul; 136(1)

8. Noutsos T, Currie BJ, Lek RA, Isbister GK (2020) Snakebite associated thrombotic microangiopathy: a systematic review of clinical features, outcomes, and evidence for interventions including plasmapheresis. PLoS Negl Trop Dis14(12): e0008936. https://doi.org/10.1371/journal. pntd.0008936

9. Longkumer et al; Journal of Venom Research | 2017 | Vol 8 | 14-18 |

10. Laxme et al; Beyond the 'big four': Venom profiling of the medically important yet neglected Indian snakes reveals disturbing antivenom deficiencies. https://doi.org/10.1371/journal.pntd.00078 99. 